\section{JTI}

JOURNAL OF

TRAUMA AND INJURY

\title{
A Peripherally Inserted Central Cathe- ter is a Safe and Reliable Alternative to Short-Term Central Venous Catheter for the Treatment of Trauma Patients
}

Received: June 12, 2019

Revised: August 19, 2019

Accepted: September 3, 2019

\section{Correspondence to}

Sang Bong Lee, M.D.

Department of Trauma and Surgical Critical Care, Pusan National University Hospital, 179 Gudeok ro, Seo-gu, Busan 49241, Korea

Tel: $+82-51-240-7369$

Fax: +82-51-247-7719

E-mail:scout79x@hanmail.net
Purpose: To determine whether a peripherally inserted central catheter (PICC) meets the goals of a low infection rate and long-term use in trauma patients.

Methods: From January 2016 to June 2018, the medical records of patients who underwent central venous catheterization at a level I trauma center were retrospectively reviewed. Data collected included age, sex, injury severity score, site of catheterization, place of catheterization (intensive care unit [ICU], emergency department, or general ward), type of catheter, length of hospital stay during catheterization, types of cultured bacteria, time to development of central line-associated bloodstream infection (CLABSI), and complications.

Results: During the study period, 333 central vein catheters (CVC) were inserted with a total of 2,626 catheter-days and 97 PICCs were placed with a total of 2,227 catheter-days. The CLABSI rate was significantly lower in the PICC group when the analysis was limited to patients for whom the catheter was changed for the first time in the ICU after CVC insertion in the ER with similar indication and catheter insertion times (18.6 vs. 10.3/1,000 catheter-days, respectively, $p<0.05)$. The median duration of catheter use was significantly longer in the PICC group than in the CVC group (16 vs. 6 days, respectively, $p<0.05)$.

Conclusions: The study results showed that the duration of catheter use was longer and the infection rate were lower in the PICC group than in the CVC group, suggesting that PICC is a safe and reliable alternative to conventional CVC.

Keywords: Catheterization, peripheral; Central venous catheters; Catheter related infections; Trauma centers 


\section{INTRODUCTION}

Central venous catheter (CVC) plays a fundamental role in the acute setting of trauma patients, as it is a reliable intravenous route for the delivery of various therapeutic fluids, as well as for hemodynamic monitoring. However, CVC is associated with an increased risk of bloodstream infection $[1,2]$, which may prolong the length of hospital stay and adversely affect patient morbidity and mortality $[3,4]$. A peripherally inserted central catheter (PICC) is a special type of CVC with insertion into a vein in the arm rather than in the neck or chest to avoid mechanical complications that may occur during conventional CVC. In addition, PICC can be used for longer periods with lower risks of bloodstream infection than conventional CVC, so its use in the hospital setting has continued to increase over the past two decades [5-7]. On the other hand, the incidence of complications is higher with PICC than with $\mathrm{CVC}$, although no significant difference in bloodstream infection rates has been reported [8,9]. Also, PICC may be a more appropriate method for outpatients than inpatients [10], although there is no general consensus. Therefore, the present study aimed to compare the rate of bloodstream infections and duration of catheter use between conventional CVC and PICC to determine whether PICC meets the goals of a lower infection rate and longterm use for trauma patients.

\section{METHODS}

The study protocol was approved by the Institutional Review Board (IRB) of Pusan National University Hospital (Busan, Korea) (IRB approval no. H-1905-031-079). From January 2016 to June 2018, the medical records of trauma patients who underwent CVC or PICC at the Pusan National University Hospital, a level I trauma center, were retrospectively reviewed. The demographic and clinical data collected included age, sex, injury severity score (ISS), site of catheterization, place of catheterization (i.e., intensive care unit [ICU], emergency department [ED], or general ward [GW]), type of catheter, length of hospital stay at the time of catheterization, duration of catheter use, type of cultured bacteria, time to development of central line-associated bloodstream infection (CLABSI), and complications.

A seven-French triple-lumen non-tunneled percutaneously inserted central catheter was used for CVC, while a five-French double-lumen percutaneously inserted catheter was used for PICC. CVC was performed by a resident of emergency medicine or an attending intensivist in the ED or ICU, while PICCs were inserted in the ICU or GW. The decision to use the subclavian vein or internal jugular vein was determined based on the experience of the practitioner and the condition of the patient. PICCs were performed by the authors at the bedside using a micro-introducer technique and portable ultrasound.

The location of the catheter tip was verified on plain chest X-rays after the procedure, while PICCs were performed with monitoring of the ipsilateral and contralateral sides of the internal jugular vein with ultrasound to prevent malposition during procedure. All CVCs and PICCs were disinfected once every 7 days for film dressing and once every 2 days for gauze dressing, and PICCs were flushed with normal saline every time fluid was replaced.

Analysis to compare bloodstream infection rates between CVCs and PICCs was limited to patients who adhered to the CVC insertion bundle (maximal sterile barrier precaution) Pusan National University Hospital (i.e., hand hygiene, wearing a cap, mask, sterile gown, sterile gloves, sterile full-body drape, $2 \%$ chlorhexidine as a disinfectant, and insertion after drying of the disinfectant). Cases of death or discharge after insertion of the CVC or PICC were excluded from analysis.

For patients with a positive blood culture, the catheter was removed and the cause of bacteremia was determined by catheter tip culture. The definition of CLABSI was in accordance with the recommendations of the National Healthcare Safety Network surveillance system [11]. The duration of catheter use was defined from the day of insertion to the day of removal. The time to infection was calculated from the day of insertion to the day of a positive blood culture. The CLABSI rate was calculated as the CLABSI event divided by the total days of catheter use, multiplied by a thousand.

\section{Statistical analysis}

All statistical analyses were conducted using IBM SPSS 
Statistics for Windows, version 21.0 (IBM Corporation, Armonk, NY, USA). The means of continuous variables were compared using Student's $t$-test, while the medians were compared using the nonparametric Mann-Whitney $U$-test. Fisher's exact test was used to compare the infection rates according to the place of insertion and the $z$-test was used to compare CLABSI rates. A probability $p$-value of $<0.05$ was considered statistically significant.

\section{RESULTS}

A total of 1,383 catheters were inserted in the present study. Of these, 111 cases of central catheterization for hemodialysis were excluded from analysis. For the remaining patients, 531 catheters were inserted in accordance with the maximal sterile barrier precaution, of which 101 patients died or were discharged from the hospital without catheter removal. A total of 430 catheters were inserted and removed in Pusan National University Hospital Level Trauma Center, which consisted of 333 CVCs and 97 PICCs.

The demographic characteristics of the two groups are presented in Table 1. There were no significant differences in age, sex, ISS, or median time from catheterization to infection between the two groups. However, there were significant differences in the median length of hospital stay at the time of insertion between the CVC and PICC groups ( $5.7 \pm 11.1$ vs. $12.9 \pm 13.8$ days, respectively, $p<0.05$ ) and the median duration of catheter use ( 7 vs. 20 days, respectively, $p<0.05$ ).

During the study period, there were 333 CVCs (sub- clavian, 316; internal jugular, 17) with a total of 2,626 catheter-days (subclavian, 2,508; internal jugular, 118). Of these, 168 were performed in the ED and 148 in the ICU. The mean and median durations of CVC were $7.9 \pm 5.8$ and 7.0 days, respectively.

In the CVC group, CLABSIs included Enterococcus faecalis $(\mathrm{n}=4)$, coagulase-negative staphylococci $(\mathrm{n}=4)$, Staphylococcus aureus $(\mathrm{n}=4)$, Clostridium celerecrescens $(\mathrm{n}=2)$, and other Gram-negative bacteria $(\mathrm{n}=10)$. In the CVC group, the CLABSI rate was 9.1/1,000 catheter-days (subclavian vein, 9.1/1,000; internal jugular vein, 8.4/1,000).

A total of 97 PICCs were inserted during the study period, with a total of 2,227 catheter-days. Of these, 90 were performed in the ICU and seven in the GW. The mean and median durations of PICC utilization were $23 \pm 17.4$ and 20 days, respectively. In the PICC group, CLABSIs included Enterococcus faecium ( $\mathrm{n}=1$ ), coagulase-negative staphylococci $(\mathrm{n}=4)$, S. aureus $(\mathrm{n}=1)$, Corynebacterium species $(n=3)$, and other Gram-negative bacteria $(n=4)$. The CLABSI rate in the PICC group was 5.8/1,000 catheter-days.

The overall CLABSI rate was higher in the PICC group than in the CVC group (13.4\% vs. $7.2 \%$, respectively, $p<0.05)$. Although this difference was not statistically significant, the CLABSI rate was lower in the PICC group than in the CVC group when converting the weighted CLABSI rate to catheter-days (5.8 vs. CVC: $9.1 / 1,000$ catheter-days, respectively, $p=0.19$ ). However, the CLABSI rate was significantly lower in the PICC group when the analysis was limited to patients for whom the catheter was changed for the first time in the ICU after CVC insertion in the ER with similar indication and catheter

Table 1. Demographic and clinical characteristics according to type of catheter

\begin{tabular}{|lccc|}
\hline Demographic/clinical characteristic & CVC $(\mathbf{n}=\mathbf{3 3 3})$ & PICC (n=97) & p-value \\
\hline Age & $55.30 \pm 17.38$ & $55.64 \pm 19.47$ & 0.88 \\
Sex, male & $253(75.9)$ & $77(79.3)$ & 0.51 \\
Length of hospital stay at catheterization (days) & $1(1-119)$ & $8(2-77)$ & $<0.05$ \\
Duration of catheter use (days) & $7(1-38)$ & $20(1-116)$ & $<0.05$ \\
Time from catheterization to infection (days) & $7(2-33)$ & $13(3-31)$ & 0.11 \\
ISS & $28(4-57)$ & $26(5-57)$ & 0.56 \\
\hline
\end{tabular}

Values are presented as mean \pm standard diviation, number (\%), or median (range).

CVC: central venous catheter, PICC: peripherally inserted central catheter, ISS: injury severity score. 
insertion times (10.3 vs. 18.6/1,000 catheter-days, respectively, $p<0.05$; Table 2). The median length of hospital stay at catheterization was 9 days (2-27) for PICC group and 6 days (1-23) for CVC group. The median duration of catheter use was significantly longer in the PICC group than in the CVC group (16 vs. 6 days, respectively, $p<0.05$; Table 3).

Although there was no significant difference in the infection rate according to the place of insertion in the PICC group, in the CVC group, the infection rate was higher when catheterization was performed in the ICU rather than in the ED (Table 4). The occurrence of complications, other than CLABSIs, according to the type of catheter is shown in Table 5. Catheter tip malposition rates were similar between the PICC and CVC groups. One $(1.0 \%)$ case of symptomatic thrombosis in PICC group and three $(0.9 \%)$ cases of pneumothorax in CVC group were noted. However, catheter dislodgement was greater in the PICC group than in the CVC group (12 [12.4\%] vs. $6[1.8 \%]$, respectively).

\section{DISCUSSION}

The CLABSI rate and duration of catheter use among trauma patients were compared between the CVC and PICC groups to determine whether PICC meets the goals of a lower infection rate and long-term use. CVC is a reliable intravenous route for the delivery of inotropic drugs, hypertonic fluids, blood products, and total parenteral nutrition to acutely ill patients, as well as for hemodynamic monitoring and blood sampling. PICC is a special type of CVC that was first introduced by Hoshal [12] and has since been used increasingly in the hospital setting [13] due to the ease of insertion into the peripheral vein, greater patient satisfaction, and improved safety $[14,15]$. In addition, PICC is considered to be associated with a lower CLABSI rate than CVC. Yamaguchi et al. [16] reported a lower CLABSI rate with PICC than with CVC (4.2\% vs. $1.4 \%$, respectively, $p<0.001)$ and overall infection risk (hazard ratio $=2.20$; 95\% confidence interval=1.05-4.61; $p=0.037$ ). Also, Gunst et al. [17] reported a lower CLABSI rate with PICC than with CVC in the surgical ICU (2.2 vs. 6.0/1,000 catheter-days, respectively, $p<0.05)$. However, it remains unclear whether PICC is beneficial for hospitalized patients, as several studies have reported that PICC is not superior to CVC due to the similarity in CLABSI rates (1.3-2.3 vs. $1.3-5.2 / 1,000$ catheter-days, respectively) $[5,18,19]$ and the difference in the duration of catheter use between the PICC and

Table 2. Difference of CLABSI rate according to the catheter type and comparison of catheter-related infection in patients of whom the catheter was changed for the first time in the ICU after CVC insertion in the ED

\begin{tabular}{|lccc|}
\hline Catheter type & Infected catheters & $\begin{array}{c}\text { CLABSI rate } \\
\text { (cases/1,000 catheter-days) }\end{array}$ & $\boldsymbol{p}$-value (vs. the CVC group) \\
\hline$C V C \rightarrow C V C(n=59)$ & $9(15.3)$ & $18.6 / 1,000$ & $<0.05$ \\
$C V C \rightarrow$ PICC $(n=32)$ & $7(21.9)$ & $10.3 / 1,000$ & \\
\hline
\end{tabular}

Values are presented as number (\%).

CLABSI: central line-associated bloodstream infection, ICU: intensive care unit, CVC: central venous catheter, ED: emergency department, PICC: peripherally inserted central catheter.

Table 3. Comparison of the duration of catheter use with or without CLABSI, and comparison of the duration of catheter use among patients of whom the catheter was changed for the first time in the ICU after CVC insertion in the ED

\begin{tabular}{|lccc|}
\hline Total patients $(\mathbf{n}=\mathbf{9 1})$ & CVC $\rightarrow$ CVC (median, days) $(\mathbf{n = 5 8 )}$ & CVC $\rightarrow$ PICC (median, days) $(\mathbf{n}=\mathbf{3 3})$ & $\mathbf{p}$-value \\
\hline CLABSls & $6(3-9)$ & $16(9-46)$ & $<0.05$ \\
No CLABSIs & $8(2-30)$ & $18(3-116)$ & $<0.05$ \\
\hline
\end{tabular}

CLABSI: central line-associated bloodstream infection, ICU: intensive care unit, CVC: central venous catheter, ED: emergency department, PICC: peripherally inserted central catheter. 
Table 4. Correlations between the incidence of CLABSI and place of catheterization

\begin{tabular}{|lcccc|}
\hline & & CLABSIs & No CLABSIs & $p$-value \\
\hline CVC & ED & $6(3.5)$ & $166(96.5)$ & $<0.05$ \\
& ICU & $17(10.6)$ & $144(89.4)$ & \\
PICC & ICU & $12(13.3)$ & $78(86.7)$ & 1.00 \\
& GW & $1(14.3)$ & $6(85.7)$ & \\
\hline
\end{tabular}

Values are presented as number (\%).

CLABSI: central line-associated bloodstream infection, CVC: central venous catheter, ED: emergency department, ICU: intensive care unit, PICC: peripherally inserted central catheter, GW: general ward.

CVC groups (19-23 vs. 13-25 days, respectively) [5,17]. The discrepancies in the CLABSI rate and duration of catheter use among institutions is thought to be related to the catheter care bundle, the compliance to guidelines by medical staff, and the department where catheterization was performed.

In the present study, there was a difference in the CLABSI rate between the PICC and CVC groups (9.1 vs. $5.8 / 1,000$ catheter-days, respectively, $p=0.19$ ). Our results were somewhat higher than those in previous studies. Although the CLABSI rate was lower in the PICC group than in the CVC group, this difference was not statistically significant. However when the analysis was limited to patients for whom the catheter was changed for the first time in the ICU after CVC insertion in the ER with similar indication and catheter insertion times, the CLABSI rate was significantly lower in the PICC group than in the CVC group (10.3 vs. 18.6/1,000 catheter-days, respectively, $p<0.05)$. Additionally, the median duration of catheter use was significantly longer in the PICC group than in the CVC group (16 vs. 6 days, respectively, $p<0.05$ ). According to these results, the CLABSI rate was lower even with a longer duration of catheter use in the PICC group, suggesting that PICC can be used safely in the ICU.

Although PICC is reported to be safe due to the lower infection rate, it is not superior to CVC because of the higher rate of complications, such as thrombosis [8]. In fact, the rate of symptomatic thrombosis with PICC is reportedly $1-25 \%$ [20] and is considered to be the most common complication of PICC. However, in this study, thrombosis occurred in only one patient $(1.0 \%)$, which is
Table 5. Catheter-related adverse effects, other than CLABSI

\begin{tabular}{|lcc|}
\hline Adverse effect & CVC & PICC \\
\hline Catheter tip malposition & $15(4.5)$ & $3(3.1)$ \\
\hline Thrombosis & $0(0)$ & $1(1.0)$ \\
Insertion site leakage/rupture & $4(1.2)$ & $2(2.1)$ \\
Catheter dislodgement & $6(1.8)$ & $12(12.4)$ \\
Pneumothorax & $3(0.9)$ & $0(0)$ \\
Insertion site redness & $5(1.5)$ & $1(1.0)$ \\
Insertion site hematoma & $1(0.3)$ & $0(0)$ \\
\hline
\end{tabular}

Values are presented as number (\%).

CLABSI: central line-associated bloodstream infection, CVC: central venous catheter, PICC: peripherally inserted central catheter.

a lower rate than those in previous studies, suggesting that PICC is sufficiently safe to replace CVC. Although not all patients were screened for thrombosis, this complication was diagnosed in symptomatic patients with the use of ultrasonography. However, 12 cases (12.4\%) of dislodgement due to movement of the patient and physical therapy occurred in the PICC group, demonstrating that such patients require close management. Pneumothorax occurred in three patients $(0.9 \%)$ in the CVC group, similar to the rate reported in previous studies [21], and all such instances were treated by closed tube thoracostomy.

The risk of PICC-associated bloodstream infection is correlated with the length of hospital stay, ICU status, and number of catheter lumen [22]. Thus, although a difference in infection rates was expected according to the place of insertion, there was no difference between procedures performed in the ICU vs. GW in patients with the PICC because of the small number of patients treated in the GW. However, there were differences between the ED and ICU in the CVC group. As a possible explanation for this difference, patients catheterized in the ICU were likely to be exposed to more infectious sources and the ICU hospitalization period was longer.

There were some limitations to this study that should be addressed. First, the results of this study should not be generalized among institutions, as this was a short-term study conducted in a single center. Although the central catheterization bundle and the central catheter care bundle are similar among most institutions, the hospital characteristics may vary from one institution to another. 
Nonetheless, similar results can be expected among institutions. Second, this was a retrospective study, which may lead to missing records of complications. The participants in this study were patients who adhered to the CVC insertion bundle (maximal barrier precaution); thus, it was not possible to consider all complications. And the checklists of the CVC insertion bundle of Pusan National University Hospital cannot be trusted completely because they were documented as interpreted by the attending nurses, rather than the physician. Third, the number of lumen differed between the PICC and CVC groups. At Pusan National University Hospital Level Trauma Center, most CVCs are initially inserted in the $\mathrm{ED}$, and resuscitation is performed in the ICU, and the CVCs that were inserted in the ED were removed. After that, the catheter was selected according to the expected period of central venous catheter use and the number of central venous catheter lumens required. However, the condition of patients requiring multi-lumen CVC was not considered to be more unstable than that of patients who received PICC. In the selection of PICC or CVC, the expected time period when the central venous line is considered necessary is more important. Rather, the patient's condition may have been in an unstable state because in the PICC group, it was expected that the patient take longer to recover. Therefore, we do not think that there is a difference in CLABSI rate according to the patient's condition. We believe that the CLABSI rate was higher in the CVC group than in the PICC group because of the greater instances of catheter hub manipulations. Therefore, studies comparing CVC and PICC of triple lumen are necessary.

\section{CONCLUSION}

In conclusion, the CLABSI rate was lower and the duration of catheter use was greater in the PICC group than in the CVC group. Fatal complications associated with PICC rarely occurred. Therefore, we believe that PICC is a safe and reliable alternative to conventional CVC for longterm maintenance via the central intravenous route in trauma patients.

\section{ACKNOWLEDGEMENTS}

This work was supported by clinical research grant from Pusan National University Hospital in 2019.

\section{REFERENCES}

1. Mermel LA, Farr BM, Sherertz RJ, Raad II, O'Grady N, Harris JS, et al. Guidelines for the management of intravascular catheter-related infections. Clin Infect Dis 2001;32:1249-72.

2. Maki DG, Kluger DM, Crnich CJ. The risk of bloodstream infection in adults with different intravascular devices: a systematic review of 200 published prospective studies. Mayo Clin Proc 2006;81:1159-71.

3. Huerta LE, Nelson GE, Stewart TG, Rice TW. Factors associated with recurrence and mortality in central line-associated bloodstream infections: a retrospective cohort study. Crit Care 2018;22:266.

4. Renaud B, Brun-Buisson C; ICU-Bacteremia Study Group. Outcomes of primary and catheter-related bacteremia. A cohort and case-control study in critically ill patients. Am J Respir Crit Care Med 2001;163:1584-90.

5. Al Raiy B, Fakih MG, Bryan-Nomides N, Hopfner D, Riegel E, Nenninger T, et al. Peripherally inserted central venous catheters in the acute care setting: a safe alternative to high-risk shortterm central venous catheters. Am J Infect Control 2010;38:14953.

6. Griffiths VR, Philpot P. Peripherally inserted central catheters (PICCs): do they have a role in the care of the critically ill patient? Intensive Crit Care Nurs 2002;18:37-47.

7. Ng PK, Ault MJ, Ellrodt AG, Maldonado L. Peripherally inserted central catheters in general medicine. Mayo Clin Proc 1997;72:225-33.

8. Safdar N, Maki DG. Risk of catheter-related bloodstream infection with peripherally inserted central venous catheters used in hospitalized patients. Chest 2005;128:489-95.

9. Govindan S, Snyder A, Flanders SA, Chopra V. Peripherally inserted central catheters in the ICU: a retrospective study of adult medical patients in 52 hospitals. Crit Care Med 2018;46:e113644.

10. Chopra V, O’Horo JC, Rogers MA, Maki DG, Safdar N. The risk of bloodstream infection associated with peripherally inserted central catheters compared with central venous catheters in 
adults: a systematic review and meta-analysis. Infect Control Hosp Epidemiol 2013;34:908-18.

11. Centers for Disease Control (CDC)/National Healthcare Safety Network (NHSN). Protocol for definition of central-line associated bloodstream infection [Internet]. Atlanta: CDC/NHSN

12. 2019 [cited cited 2019 Jun 7]. Available from: https://www.cdc. gov/nhsn/PDFs/pscManual/4PSC_CLABScurrent.pdf.

13. Hoshal VL Jr. Total intravenous nutrition with peripherally inserted silicone elastomer central venous catheters. Arch surg 1975;110:644-6.

14. Tejedor SC, Tong D, Stein J, Payne C, Dressler D, Xue W, et al. Temporary central venous catheter utilization patterns in a large tertiary care center: tracking the "idle central venous catheter". Infect Control Hosp Epidemiol 2012;33:50-7.

15. Todd J, Hammond P. Choice and use of peripherally inserted central catheters by nurses. Prof Nurse 2004;19:493-7.

16. Schwengel DA, McGready J, Berenholtz SM, Kozlowski LJ, Nichols DG, Yaster M. Peripherally inserted central catheters: a randomized, controlled, prospective trial in pediatric surgical patients. Anesth Analg 2004;99:1038-43.

17. Yamaguchi RS, Noritomi DT, Degaspare NV, Muñoz GOC, Porto APM, Costa SF, et al. Peripherally inserted central catheters are associated with lower risk of bloodstream infection compared with central venous catheters in paediatric intensive care patients: a propensity-adjusted analysis. Intensive Care Med 2017;43:1097-104.

18. Gunst M, Matsushima K, Vanek S, Gunst R, Shafi S, Frankel H. Peripherally inserted central catheters may lower the incidence of catheter-related blood stream infections in patients in surgical intensive care units. Surg Infect (Larchmt) 2011;12:279-82.

19. Gahlot R, Nigam C, Kumar V, Yadav G, Anupurba S. Catheterrelated bloodstream infections. Int J Crit Illn Inj Sci 2014;4:162-7.

20. Skiest DJ, Abbott M, Keiser P. Peripherally inserted central catheters in patients with AIDS are associated with a low infection rate. Clin Infect Dis 2000;30:949-52.

21. Liu Y, GAO Y, Wei L, Chen W, Ma X, Song L. Peripherally inserted central catheter thrombosis incidence and risk factors in cancer patients: a double-center prospective investigation. Ther Clin Risk Manag 2015;11:153-60.

22. Kornbau C, Lee KC, Hughes GD, Firstenberg MS. Central line complication. Int J Crit illn Inj Sci 2015;5:170-8.

23. Chopra V, Ratz D, Kuhn L, Lopus T, Chenoweth C, Krein S. PICC-associated bloodstream infections: prevalence, patterns, and predictors. Am J Med 2014;127:319-28. 